\title{
Probabilistic Multi-shape Segmentation of Knee Extensor and Flexor Muscles
}

\author{
Shawn Andrews ${ }^{1}$, Ghassan Hamarneh ${ }^{1}$, Azadeh Yazdanpanah ${ }^{1}$, \\ Bahareh HajGhanbari ${ }^{2}$, and W. Darlene Reid ${ }^{2}$ \\ 1 Medical Image Analysis Lab, Simon Fraser University, Canada \\ $\{$ sda56, hamarneh, aya18\}@sfu.ca \\ 2 Department of Physical Therapy, University of British Columbia, Canada \\ wdreid@mail.ubc.ca, baharehg@interchange.ubc.ca
}

\begin{abstract}
Patients with chronic obstructive pulmonary disease (COPD) often exhibit skeletal muscle weakness in lower limbs. Analysis of the shapes and sizes of these muscles can lead to more effective therapy. Unfortunately, segmenting these muscles from one another is a challenging task due to a lack of image information in many areas. We present a fully automatic segmentation method that overcomes the inherent difficulties of this problem to accurately segment the different muscles. Our method enforces a multi-region shape prior on the segmentation to ensure feasibility and provides an energy minimizing probabilistic segmentation that indicates areas of uncertainty. Our experiments on 3D MRI datasets yield an average Dice similarity coefficient of $0.92 \pm 0.03$ with the ground truth.
\end{abstract}

\section{Introduction}

In patients with chronic obstructive pulmonary disease (COPD), skeletal muscle weakness is common [9, 1]. Lower limb muscles are often the most affected due to lack of use [1]. The skeletal muscle weakness related to COPD may be associated with the loss of muscle mass [1]. Reduced muscle mass is among several factors that lead to reduced force production, also including changes in the muscle contractile apparatus and neuromuscular activation. The relative effects of these factors are unknown [9]. Thus, size and shape measurements are required to study the contribution of muscle mass reduction to force loss. Recently [11], a non-uniform distribution of atrophy and size changes was found across knee extensors and flexors in patients with COPD, which may be reflective of localized factors such as denervation, limited recruitment, or atrophy of specific muscle fibers, rather than systemic factors contributing to muscle atrophy.

Magnetic resonance imaging (MRI) can be used to distinguish muscle from the surrounding regions, and can generate multiple image slices from which volume and shape properties of individual muscle can be estimated [6]. Information regarding the specific muscles or muscle regions that are most atrophied together with functional assessment will enable therapeutic interventions to be targeted to the affected regions [11,14] rather than the prescription of a generalized approach that may prove ineffectual.

An important precursor to any volume measurement or shape analysis is segmentation. Manual segmentation in 3D medical images is extremely time consuming, tedious, 

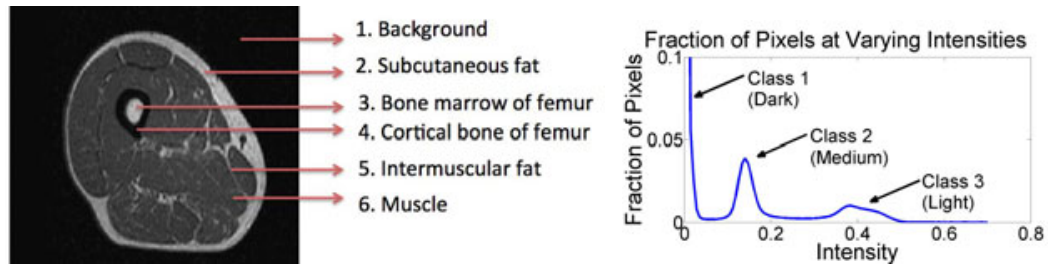

Fig. 1. Regions in the thigh, and the intensity profiles of the thigh MRIs. We see the background and cortical bone are dark, the muscle is medium, and the fat and bone marrow are light.

and suffers from inter- and intra-operator variability. Highly automated segmentation is important for studies involving a large cohort of subjects to reduce manual labour and variability, and to improve the efficiency in analyzing large groups of data.

Segmentation of thigh muscles is a challenging task, especially segmenting all 11 knee extensor and flexor muscles. As seen in Fig. 1, all these muscles have almost identical voxel intensities and weak inter-muscular image gradients. Similar muscle segmentation problems have been tackled previously using innovative techniques [15, 8. 7], but to the best of our knowledge there exists no work creating a fully automatic method for segmenting all 11 muscles from an MRI.

In this paper we present a segmentation method tailored to this specific problem. Given the difficulty of the problem, mainly due to the lack of image information in many regions, we generate a probabilistic segmentation in order to express confidence, or lack thereof, in these regions. In order to overcome the limited image information available, we will enforce a principal component analysis (PCA) based shape prior, constructed from training data, similar to [3]. Before PCA is performed on the probabilistic training segmentations, an anatomy-driven spatial alignment is performed and the isometric logratio (ILR) transform [2,5] is applied to the training segmentations, thus moving them to a vector space to facilitate algebraic manipulation intrinsic to the probability simplex. A convex energy-minimization formulation is adopted that guarantees globally optimal solution insensitive to initialization.

\section{Method}

\subsection{The ILR Transform}

A probabilistic segmentation can be represented as a function $q: \Omega \rightarrow \mathbb{S}^{R}$, where $\Omega$ is the image domain and $\mathbb{S}^{R}$ is the simplex of all valid $R=12$ length probability vectors (11 muscles and background). If we define a function $\phi: \mathbb{S}^{R} \rightarrow \mathbb{R}^{R-1}$, bijectively mapping probability vectors to a vector of real numbers, then we can also represent probabilistic segmentations by another function $\eta=\phi \circ q: \Omega \rightarrow \mathbb{R}^{R-1}$. Using this representation, the space of segmentations, referred to as ILR space, is closed under linear combinations. This allows us to perform PCA on training segmentations without the need for constraints to ensure our results have valid probabilities, summing to unity.

We define $\phi$ as the ILR transform [2,5]. In [5], the Aitchison inner product $\langle p, q\rangle_{a}$ and Aitchison distance metric $d_{a}(p, q)$ are defined for $p, q \in \mathbb{S}^{R}$. With the Aitchison 
inner product, a basis for $\mathbb{S}^{R}$ can be defined, denoted, $B_{E}=\left\{\mathbf{e}_{1}, \ldots, \mathbf{e}_{R-1}\right\}$. There are many choices for $B_{E}$ and some can be found in [5]; the exact choice of $B_{E}$ does not affect our method. Given $B_{E}$, the ILR transform of $p \in \mathbb{S}^{R}$ is defined as:

$$
\phi(p)=\left[\left\langle p, \mathbf{e}_{1}\right\rangle_{a}, \ldots,\left\langle p, \mathbf{e}_{R-1}\right\rangle_{a}\right] \in \mathbb{R}^{R-1},
$$

i.e. the projection of $p$ onto the basis $B_{E}$. While there are several possible functions that map $\mathbb{S}^{R}$ to a Euclidean space [13], we choose the ILR transform due to the fact that it is bijective (thus easily invertible) and an isometry. That is, for $p, q \in \mathbb{S}^{R}, d_{a}(p, q)=$ $d(\phi(p), \phi(q))$, where $d(\cdot, \cdot)$ is the standard Euclidean distance. Finally, we discretize $\Omega$ to $n$ voxels and represent segmentations $\eta$ as a vectors of $n(R-1)$ reals. We perform PCA on segmentations in this form to create our shape space.

\subsection{The Shape Space}

A strong shape prior that considers all of the muscles simultaneously is appropriate for this problem as the shape and relative locations of the muscles are highly correlated from one image to another. Furthermore, as the muscles are adjacent to each other along much of their boundaries, the shapes of different muscles in the same image are correlated. We construct such a shape prior by identifying a subspace of possible segmentations that corresponds to feasible thigh muscle segmentations and force our segmentation to lie in this space. To do this, we perform PCA on vectors $\left\{\hat{\eta}_{1}, \ldots, \hat{\eta}_{N}\right\}$ in $\mathbb{R}^{n(R-1)}$, corresponding to training probabilistic ground truth (GT) segmentations.

We define $\eta_{0}$ as the mean of the training GTs, $\Gamma$ as the $(n(R-1)) \times k$ matrix whose columns correspond to the PCA eigenmodes of greatest variance, and $\Lambda$ as the $k \times k$ diagonal matrix whose diagonal elements are the eigenvalues (variances) of the eigenmodes of $\Gamma$. We parameterize segmentations in our shape space by $\gamma \in \mathbb{R}^{k}$, so a segmentation is represented as

$$
\eta(\gamma)=\eta_{0}+\Gamma .
$$

$\eta(\gamma)$ is linear in $\gamma$, so any energy functions that are convex in $\eta$ will be convex in $\gamma$.

\subsection{Image Alignment}

In order to construct the shape space in Section 2.2, the thigh images must first be roughly aligned. To do this, we take advantage of some specific anatomical and image

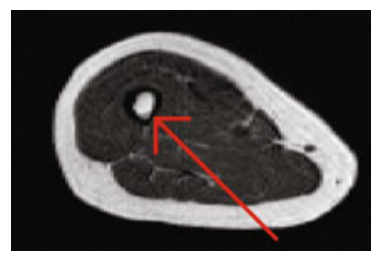

Bone in a Slice

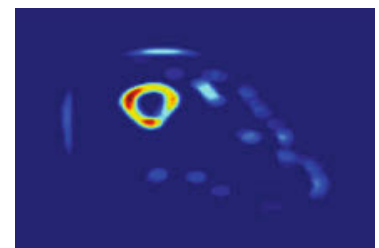

Bone Indicator

Fig. 2. A slice of a thigh image with the location of the bone highlighted (left, red arrow) and the locations of interfaces between medium and dark regions, indicating the boundary of the femur (right) 
features. To bring the images into rough alignment, for each image we note that the images exhibit 3 distinctive intensity classes, referred to as "dark", "medium", and "light". From Fig. 1 we see that background and cortical femur bone are dark, the muscles are medium, and the bone marrow and fat are light. We use these strong intensity priors to separate the muscles from the background and then crop the images accordingly.

To further align the images, we note the femur bone running through the middle of the thigh muscles follows a roughly straight vector through each image. As seen in Fig. 2, the bone is characterized by an interface between dark and medium intensity voxels, and such an interface occurs infrequently elsewhere in the image. For a given image, we estimate the vector along which the bone travels by finding its center, $B(z)=\left(x_{B}(z), y_{B}(z)\right)$, in as many slices $(z)$ as possible. We then fit a linear function $f(z)=\left(a_{x} z+b_{x}, a_{y} z+b_{y}\right)$ to estimate the coordinate of the centre of the bone in each slice. Using $f(z)$, we translate each slice in each image so that the femur follows the same vector in each image. Finally, we rescale the images isotropically so that their foregrounds are roughly the same size. This method is simple and quick, and we found it to provide an adequate, anatomy-driven alignment for construction of the shape space. A more complex alignment would unnecessarily shift work from our efficient convex segmentation method to an expensive non-convex registration scheme.

\subsection{Energy Construction}

With the images aligned and a shape space constructed, we will use image information to construct a strictly convex energy function over the shape space. The energy will be constructed such that viable segmentations yield lower energy values, and since it is strictly convex, the minimization of our energy will require no initialization.

As seen in Fig. 1) the different muscle regions have almost identical intensity distributions and textures, but the non-muscle region (fat and bone) has significantly different intensities. Thus, we create a probabilistic segmentation $q_{B G}$, where voxels with light or dark intensities are assigned to the non-muscle region with probability 1 and voxels with medium intensities are assigned probability $\frac{1}{11}$ for each of the 11 muscle regions. We then define an energy term:

$$
E_{B G}(\gamma)=d\left(\eta_{B G}, \eta(\gamma)\right)^{2}
$$

$E_{B G}(\gamma)$ is convex in $\eta$, and thus convex in $\gamma$, and encourages $\eta$ to give high probability to the non-muscle region in appropriate places.

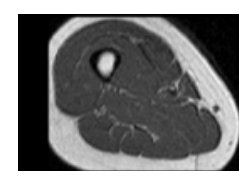

Slice Intensities

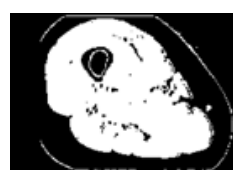

Thresholded Intensities

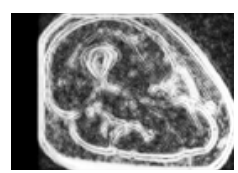

Curvature: $c$

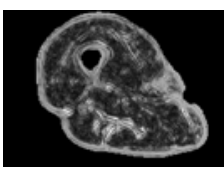

Curvature: $\bar{c}$

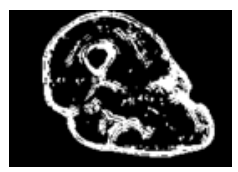

Boundary: $h$

Fig. 3. Extracted image information. From left to right, we see for a thigh slice: the intensity values; the approximation of the muscle regions extracted by intensity thresholding; the curvature values $c$ and $\bar{c}$; and the boundary estimate $h$. 
While the muscle regions are largely homogeneous in intensity, weak intensity gradients appear along the inter-muscular region boundaries (Fig. 3). The boundaries between muscle regions are characterized by a thin sheet of somewhat higher intensity fat voxels with lower intensity muscle voxels on either side, and therefore boundary voxels exhibit large curvature in the intensity in the direction perpendicular to the boundary. Thus we construct the Hessian of the intensity at each voxel $x$ and find its eigenvalues, from largest to smallest magnitude, $c_{1}(x), c_{2}(x)$, and $c_{3}(x)$, and the corresponding normalized eigenvectors $v_{1}(x), v_{2}(x)$, and $v_{3}(x) . c_{1}(x)$ and $v_{1}(x)$ correspond to the maximum curvature and its direction. For voxels on these sheet boundaries, we expect $c_{1}(x) \gg c_{2}(x), c_{3}(x)[4]$. Thus, we take $c(x)=\sqrt{c_{1}(x)^{2} /\left(c_{1}(x)^{2}+c_{2}(x)^{2}+c_{3}(x)^{2}\right)}$ as a muscle boundary indicator at $x$ (Fig. 3).

Once we have found $c$, we further filter out noise. To do this, we note that $v_{1}(x)$ for $x$ on an intermuscular sheet of fat will be similar to $v_{1}(y)$ for a voxel $y$ neighboring $x$ within the sheet, and that the span of $v_{2}(x)$ and $v_{3}(x)$ will approximate the plane of the boundary. Thus we apply a specialized bilateral filter to $c(x)$, smoothing $c(x)$ with nearby voxels on the boundary, weighted by the dot product between the their directions of maximal curvature. Specifically, if $y(\alpha, \beta)=x+\alpha v_{2}(x)+\beta v_{3}(x)$ then

$$
\bar{c}(x)=\sum_{\alpha=-2}^{2} \sum_{\beta=-2}^{2} \frac{\left|\left\langle v_{1}(x), v_{1}(y(\alpha, \beta))\right\rangle\right|}{\sqrt{1+\alpha^{2}+\beta^{2}}} c(y(\alpha, \beta)) .
$$

The boundary between the dark background and light subcutaneous fat does not help in the segmentation, so it is identified (based on intensity) and removed from $\bar{c}$ (Fig. 3).

We construct $\bar{c}$ for each training image and find $c_{\min }$ and $c_{\max }$ such that the set $H=\left\{x \mid c_{\min } \leq \bar{c}(x) \leq c_{\max }\right\}$ has the maximal Dice similarity coefficient (DSC) with the true set of boundary voxels (derived from the training GTs). Given a novel image, we construct $\bar{c}$, threshold to find $H$, and define $h: \Omega \rightarrow\{0,1\}$ as the indicator function for $H$ (Fig. 3). We use the boundary based energy term

$$
E_{B D Y}(\gamma)=\sum_{r=1}^{R-1} \sum_{x \in \Omega}(1-h(x))\left|\nabla_{x} \eta\right|^{2} .
$$

$E_{B D Y}$ allows the segmentation to have large gradient only on voxels deemed likely to be part of a boundary, otherwise the gradient is penalized. Note once again that $E_{B D Y}$ is convex in $\eta$ and thus in $\gamma$. Also note that $\nabla_{x} \eta$ measures the rate of change between the probabilities of neighboring voxels measured via the Aitchison distance.

Our final energy term enforces a Mahalanobis type penalty on $\gamma$, allowing components corresponding to eigenmodes of greater variance to vary more:

$$
E_{\text {Shape }}(\gamma)=\gamma^{T} \Lambda^{-1} \gamma
$$

Our energy is a linear combination of the convex energy terms (3), (5), and (6):

$$
E(\gamma)=\lambda_{1} E_{B G}(\gamma)+\lambda_{2} E_{B D Y}(\gamma)+\lambda_{3} E_{\text {Shape }}(\gamma)
$$

Once our energy is constructed, we segment a novel image by finding $\gamma_{\min }=$ $\operatorname{argmin}_{\gamma} E(\gamma)$, and taking $q_{\text {seg }}=\phi^{-1}\left(\eta_{0}+\Gamma \gamma_{\text {min }}\right)$ as our final probabilistic segmentation. 


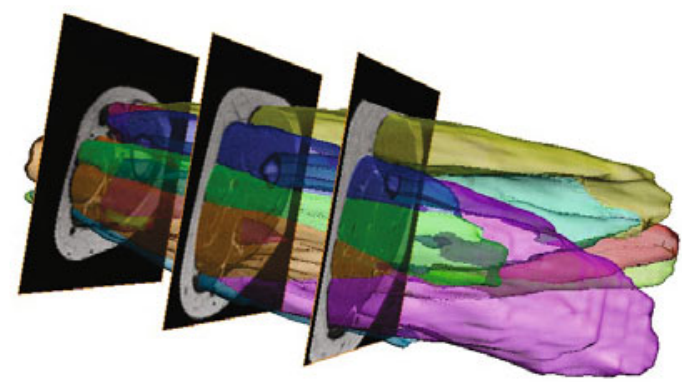

Fig. 4. (Color Figure) The resulting segmentation of our method overlaid on several image slices. Note the matching of the segmentation to the image boundaries.

\section{Results}

We report results on 3D MRI data from 40 subjects including 20 healthy subjects (11 women, age: $64.4 \pm 8.1$ years) and 20 moderate to severe COPD patients (11 women, age: $68 \pm 10$ years). A $1.5 \mathrm{~T}$ MRI scanner acquired $5 \mathrm{~mm}$-thick axial T1-weighted magnetic resonance (echo time, $8 \mathrm{~ms}$; repetition time, $650 \mathrm{~ms}$ ) with a $40 \mathrm{~cm}^{2}$ field of view and a $512 \times 384$ pixel matrix (in-plane resolution, $0.78 \times 1.78 \mathrm{~mm}$ ). The MRI scan yielded a total of approximately 100 slices for each participant. To create the GT's, these images were manually segmented and each pixel was given a probability approaching 1 for the region it was assigned to.

We cropped the images down to a $175 \times 175 \times 85$ volume enclosing the thigh, and aligned the images using the method described in Section 2.3 . We test our method using leave-one-out validation (removing one of the volumes and training a shape space using the remaining 39 volumes). Following [12], we increase the dimensions of the shape space by deforming each of the 39 training GT segmentations 3 times, giving 156 segmentations on which to train a shape space. Adding such deformed volumes to the training data will increase the expressibility of the shape space. We perform PCA on the training segmentations, finding the $k=100$ eigenmodes of greatest variance.

We measured the success of our algorithm using the DSC between the segmentation we generate, thresholded to non-probabilistic, and the corresponding GT. In Fig. 4, we
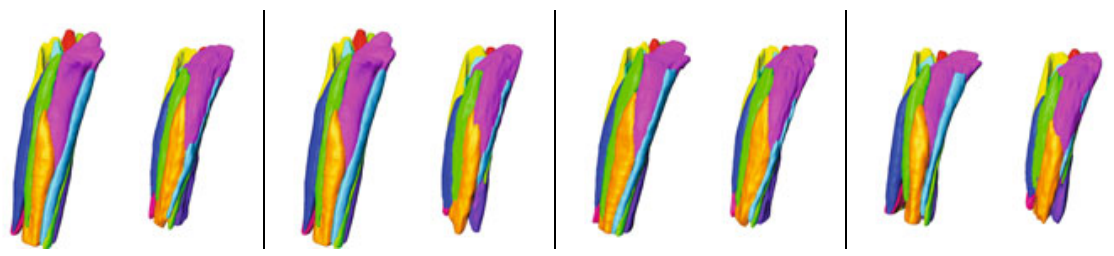

Fig. 5. (Color Figure) A comparison between several of the GT segmentations (left side of each pair) and the segmentations generated by our method (right side of each pair). The left two pairs are from COPD patients and the right two pairs are from non-COPD patients. DSC scores for each pair are $0.94,0.91,0.95$, and 0.93 . 


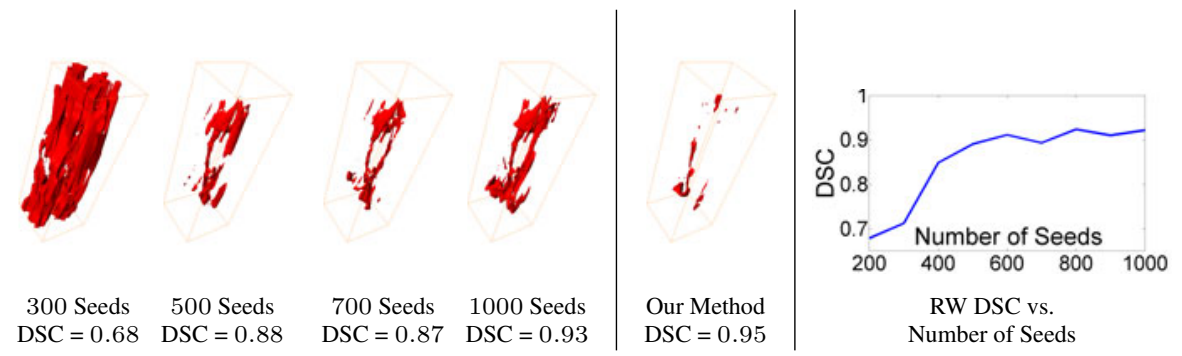

Fig. 6. The areas of mis-segmentation by the RW algorithm with varying numbers of randomly selected seeds (left) and our algorithm (center), along with a graph showing how the average DSC of RW changes with the number of seeds used (right)

see a segmentation generated by our method overlaid against slices of the image. We note our energy successfully matches the segmentation to boundaries in the image. We see examples of our segmentations compared to the GTs in Fig. 5, with DSC scores reported. We achieved an average DSC values of $0.92 \pm 0.03$ across all images.

To demonstrate the difficulty of the problem and the necessity of our shape prior, we segmented the thigh volumes using the popular random walker (RW) [10] algorithm, which also produces multi-region probabilistic segmentations and achieves the global minimum of its energy, but does not include a shape prior. RW requires seed voxels from each region to perform a segmentation, so we used the GT segmentations to provide between 200 and 1000 seeds, randomly sampled throughout the volume. In Fig. 6, we see the areas where the GT disagreed with our method and the various RW versions. RW only begins performing comparably to our method when the number of seed voxels exceeds 1000. In Fig. 6, we also see how the RW accuracy increases with the number of seeds provided. Note that without the GTs to provide automatic seeds, all seeds would have to be manually input, which would be very time consuming for a user. Also note that when 700 seeds were provided, the resulting DSC in Fig. 6 was worse than when only 500 seeds were provided, showing how the semi-automatic RW algorithm is dependent on seeding quality as well as quantity. In contrast, our method is strictly convex and fully automatic, not depending on user input.

\section{Conclusion}

The results of this work will be critical in clinical research aiming to determine factors affecting muscle structure and function in chronic conditions such as COPD. The methods proposed in this work will assist in more accurate measurement of muscle periphery and other size measures. Moreover it will provide researchers and clinicians with a time-efficient way to diagnose muscle anomalies associated with chronic conditions. For future work, we will explore projecting training GTs onto the shape space so that analysis of their eigenmode weights can be performed. These weights could both provide information about the adverse effects of COPD on the thigh muscle structure and help identify the disease in novel images. Our future work will also include exploring more advanced alignment techniques and comparing to other segmentation methods. 


\section{References}

1. Bernard, S., LeBlanc, P., Whittom, F., Carrier, G., Jobin, J., Belleau, R., Maltais, F.: Peripheral muscle weakness in patients with chronic obstructive pulmonary disease. Am. J. Respir. Crit. Care Med. 158(2), 629-634 (1998)

2. Changizi, N., Hamarneh, G.: Probabilistic multi-shape representation using an isometric logratio mapping. In: Jiang, T., Navab, N., Pluim, J., Viergever, M. (eds.) MICCAI 2010. LNCS, vol. 6363, pp. 563-570. Springer, Heidelberg (2010)

3. Cremers, D., Schmidt, F., Barthel, F.: Shape priors in variational image segmentation: Convexity, lipschitz continuity and globally optimal solutions. In: CVPR 2008, pp. 1-6. IEEE, Los Alamitos (2008)

4. Descoteaux, M., Audette, M., Chinzei, K., Siddiqi, K.: Bone enhancement filtering: Application to sinus bone segmentation and simulation of pituitary surgery. Comput. Aided Surg. 11(5), 247-255 (2006)

5. Egozcue, J., Pawlowsky-Glahn, V., Mateu-Figueras, G., Barceló-Vidal, C.: Isometric logratio transformations for compositional data analysis. Mathematical Geology 35, 279-300 (2003)

6. Engstrom, C., Loeb, G., Reid, J., Forrest, W., Avruch, L.: Morphometry of the human thigh muscles. A comparison between anatomical sections and computer tomographic and magnetic resonance images. Journal of Anatomy 176, 139-156 (1991)

7. Essafi, S., Langs, G., Deux, J.F., Rahmouni, A., Bassez, G., Paragios, N.: Wavelet-driven knowledge-based mri calf muscle segmentation. In: Proceedings of the Sixth IEEE International Conference on Symposium on Biomedical Imaging: From Nano to Macro, ISBI 2009, pp. 225-228. IEEE Press, Los Alamitos (2009)

8. Gilles, B., Magnenat-Thalmann, N.: Musculoskeletal mri segmentation using multiresolution simplex meshes with medial representations. Medical Image Analysis 14(3), 291-302 (2010)

9. Gosselink, R., Troosters, T., Decramer, M.: Peripheral muscle weakness contributes to exercise limitation in COPD. Am. J. Respir. Crit. Care Med. 153(3), 976-980 (1996)

10. Grady, L.: Multilabel Random Walker Image Segmentation Using Prior Models. In: CVPR 2005, pp. 763-770. IEEE, Los Alamitos (2005)

11. HajGhanbari, B., Hamarneh, G., Changizi, N., Ward, A., Reid, W.: MRI-Based 3D Shape Analysis of Thigh Muscles: Patients with Chronic Obstructive Pulmonary Disease Versus Healthy Adults. Acad. Radiol. 18(2), 155-166 (2011)

12. Hamarneh, G., Jassi, P., Tang, L.: Simulation of ground-truth validation data via physicallyand statistically-based warps. In: Metaxas, D., Axel, L., Fichtinger, G., Székely, G. (eds.) MICCAI 2008, Part I. LNCS, vol. 5241, pp. 459-467. Springer, Heidelberg (2008)

13. Pohl, K.M., Fisher, J., Bouix, S., Shenton, M., McCarley, R.W., Grimson, W.E.L., Kikinis, R., Wells, W.M.: Using the logarithm of odds to define a vector space on probabilistic atlases. Medical Image Analysis 11(5), 465-477 (2007)

14. Tracy, B., Ivey, F.M., Metter, E.J., Fleg, J.L., Siegel, E.L., Hurley, B.F.: A more efficient magnetic resonance imaging-based strategy for measuring quadriceps muscle volume. Med. Sci. Sports Exerc. 35(3), 425-433 (2003)

15. Wang, C., Teboul, O., Michel, F., Essafi, S., Paragios, N.: 3d knowledge-based segmentation using pose-invariant higher-order graphs. In: Jiang, T., Navab, N., Pluim, J.P.W., Viergever, M.A. (eds.) MICCAI 2010. LNCS, vol. 6363, pp. 189-196. Springer, Heidelberg (2010) 